\title{
Cyclical Properties of a Real Business Cycle Model
}

\author{
Paul Söderlind* \\ Institute for International Economic Studies \\ Stockholm University \\ S-106 91 Stockholm, Sweden
}

First version: October 1990

This version: August 1993

\begin{abstract}
This paper tests the well-known real business cycle model of Kydland and Prescott (1988), using spectral methods for linear filters. Model spectra, coherencies, phase shifts, and correlations are tested against US postwar data using both asymptotic and small-sample distributions. Compared with the model, the data has shorter business cycles, smaller comovements of different macro variables, and less of a leading role for output.
\end{abstract}

*I am grateful to seminar participants at IIES and Princeton University; John Campbell, Gregory Chow, Nils Gottfries, Mats Kinnvall, Harald Lang, Robin Lumsdaine, Petter Lundvik, Torsten Persson, Ken Rogoff, Lars E.O. Svensson, Anders Vredin and two referees for comments; Mark Bils for comments and data; and Molly Åkerlund for editorial assistance. 


\section{Introduction}

The most common way to evaluate the fit of real business cycle models is to compare correlations from model simulations with those of the data. There are at least two problems associated with this procedure. First, from the statistical perspective it is unfortunate that the comparison is seldom made in terms of statistical inference; in other words, are the differences really significant? Second, from the economic perspective it is not entirely clear how these few correlations map into business cycles - often thought of as being of 3 to 8 years duration.

This paper tries to assess the cyclical properties of the model of Kydland and Prescott (1988) in a way that suffers less from this critique. Model spectra, coherencies, phase shifts, and correlations are tested against US postwar data using both asymptotic and small-sample distributions. The use of frequency domain statistics, which are not common in the real business cycle context ${ }^{1}$, allows us to detect and test certain features of the model much more easily than in the time domain.

The results indicate that the business cycles in the model are somewhat too long, and are of insufficient magnitude at the traditional business cycle frequencies. The model also predicts comovements of different macro variables that are too high, and an exaggerated role for output as a driving force in the business cycle.

\section{A Brief Summary of Kydland and Prescott's Model}

The model in Kydland and Presscott (1988) is a stochastic neo-classical intertemporal model of a competitive and closed economy. It has five distinguishing features. First, lags of leisure enter the period subutility function in order to increase intertemporal substitution of labor supply. Second, there are increasing returns to scale in production, aimed at capturing a capacity utilization effect. Third, inventories are modeled in order to capture their well-known importance for the business cycle. Fourth, investments are transformed into productive capital with a three-quarter delay ("time-to-build"). This increases the degree of endogenous persistence in output and reduces its correlation with the capital stock. Fifth, there are three productivity disturbances: a persistent component which follows $z_{1 t+1}=\rho z_{1 t}+\xi_{t+1}$ with $\rho=0.95$, a transitory component, and a measurement error. The transitory productivity component generates short-run noise without contributing to the long-run cycles which, among other things, increases the volatility of output relative to consumption. The measurement error is present at the beginning of each period when agents make investment

\footnotetext{
${ }^{1}$ However, see Altuğ (1989), Watson (1993) and Cogley and Nason (1992) for applications to RBC models.
} 
and labor supply decisions, but not at the end of the period when consumption decisions are made. The main effect of this measurement error is to increase the volatility of inventories, which play a buffer stock role.

\section{Spectra of Kydland and Prescott's Model}

The solution to the approximate (quadratic) optimization problem gives linear decision rules in terms of the disturbances and other state variables. The only remaining non-linearity is in the production function, which I linearize in order to get a completely linear system. Rearranging the decision rules in the form of a vector moving average of the three disturbances, that is, $y_{t}=B(\mathrm{~L}) z_{t}$, where $z_{t}$ is the $3 \times 1$ vector of disturbances, provides a very convenient way to calculate the spectral density function of the model.

The diagonal matrix of spectral density at frequency $\lambda$ for the three basic disturbances (an $\mathrm{AR}(1)$ and two white noise processes, which are independent) is

$$
f^{z}(\lambda)=\operatorname{diag}\left(\frac{\sigma_{1}^{2} / 2 \pi}{1+\rho^{2}-2 \rho \cos (\lambda)}, \sigma_{2}^{2} / 2 \pi, \sigma_{3}^{2} / 2 \pi\right),
$$

where $\sigma_{i}^{2}$ is the variance of the $i^{t h}$ innovation. The spectral density function for the model variables is then

$$
f^{M}(\lambda)=B\left(e^{-i \lambda}\right) f^{z}(\lambda) B\left(e^{i \lambda}\right)^{\prime},
$$

where $\mathrm{B}\left(e^{-i \lambda}\right)$ is the transfer function matrix, with the $i j^{t h}$ element calculated as the Fourier transform of the MA coefficients on the $j^{\text {th }}$ disturbance for the $i^{\text {th }}$ model variable. ${ }^{2}$

Kydland and Prescott (1988) choose to filter both data and simulation results with the Hodrick-Prescott (H-P) filter. Since samples are of finite lengths, this filter will not be exactly the same for all observations. Here, we use the asymptotic filter discussed in King and Rebelo (1993), which has constant coefficients, as an approximation. This approximation is very good for observations not very close to the start or the end of the sample. ${ }^{3}$ The transfer function $B^{H P}(\lambda)$ of this filter is

$$
B^{H P}(\lambda)=\frac{16 \sin ^{4}(\lambda / 2)}{1 / 1600+16 \sin ^{4}(\lambda / 2)},
$$

which is shown (squared) in Figure 1 (thin solid curve). As reference, 3 and 8 year cycles are marked by vertical lines. This filter blocks out 16 year cycles almost completely, 8 year cycles are halved (in terms of variance), while 4 year cycles are left virtually unchanged.

\footnotetext{
${ }^{2}$ See, for instance, Priestley (1981) for a discussion of transfer functions of linear filters.

${ }^{3}$ The finite sample version shows considerable phase shift close to the beginning and end of the sample. In practice, this can displace the business cycle by a year or more in standard macro series, which makes the H-P filter a bad choice for "real-time" applications.
} 
Figure 1 also shows the normalized spectral density of the unfiltered (thick solid curve) and H-P filtered (dashed curve) AR(1) productivity disturbance. As illustrated, the effect of the filter is to make the traditional business cycle frequencies relatively more important. In fact, it can be shown that the spectral density maximum for the H-P filtered $\mathrm{AR}(1)$ is at

$$
\begin{aligned}
& \lambda^{\max }=\arccos \left(\frac{-1+160 w^{\frac{1}{3}}-w^{\frac{2}{3}}+i 3^{\frac{1}{2}}\left(w^{\frac{2}{3}}-1\right)}{160 w^{\frac{1}{3}}}\right), \text { where } \\
& w \quad=160-80\left(\frac{1}{\rho}+\rho\right)+\left(25599-25600\left(\frac{1}{\rho}+\rho\right)+6400\left(\frac{1}{\rho}+\rho\right)^{2}\right)^{\frac{1}{2}} .
\end{aligned}
$$

With $\rho=0.95$, as in Figure 1 , this gives a maximum at 7 years and 5 months.

The spectral density function for the H-P filtered model variables is

$$
f(\lambda)=\left(B^{H P}(\lambda)\right)^{2} f^{M}(\lambda)=\left(B^{H P}(\lambda)\right)^{2} B\left(e^{-i \lambda}\right) f^{z}(\lambda) B\left(e^{i \lambda}\right)^{\prime} .
$$

Transformations of the elements in $f(\lambda)$ give spectra, coherencies, phase shifts, and also correlations. These will be discussed and compared with empirical estimates in Section 4.

Serious criticism of the H-P filter, related to the effect illustrated in Figure 1, has been raised by, among others, Singleton (1988), Cogley and Nason (1992), and Harvey and Jaeger (1993). ${ }^{4}$ It is therefore useful to consider statistics which are invariant to linear filters. This is the case for coherencies and phase shifts, but not for spectra or correlations.

\section{Empirical Findings}

\subsection{Data and testing approach}

This section compares estimated spectra, coherencies, phase shifts and correlations for the United States with model predictions. The data used are seasonally adjusted quarterly data for the United States for the period 1949:2-1984:3 (143 observations). ${ }^{5}$ All series have been filtered with the H-P filter.

The testing approach has the flavor of testing the null hypothesis that data is a realization of the model economy. However, a scalar test statistic is not constructed. Instead, each variable and frequency is tested separately, which may help us to understand how and where the model fails. The main tools are the theoretical asymptotic $90 \%$ confidence intervals. These are constructed from

\footnotetext{
${ }^{4}$ Another perspective is that the filtered model results could also be obtained by filtering the exogenous disturbances before simulating, while still using the same prediction and decision rules. These rules are clearly not optimal when confronted with filtered disturbances.

${ }^{5}$ The data is measured in 1982 prices, and is taken from Citibase, except for the consumption series, which is from Christiano (1988). Output is GNP; consumption is expenditures on nondurables and services, plus a flow of services from durables; investment is gross private domestic investment; and inventory stock is farm plus nonfarm inventory stock.
} 
the theoretical spectra, but take the empirical estimation method into account. ${ }^{6}$ The confidence intervals are the thin solid curves in Figures 2a-d, Figures 3a-c and Figures 4a-c. The empirical point estimates are the dashed curves.

We know little about the small-sample properties of spectral density estimators. Therefore, small-sample distributions have been generated by simulating the model over 143 quarters 3000 times. The small panels inserted in Figures 2a-d and shown in Figure $3 d$ and Figure $4 d$ show at which significance level, according to the simulated distribution, the fit of the model can be rejected. The $10 \%$ level is marked with horizontal lines.

\subsection{Results}

The model spectra before H-P filtering look pretty much like the unfiltered AR(1) shown in Figure 1, but with a small peak around three quarters. The model spectra after H-P filtering are located approximately in the middle of the confidence intervals in Figures 2a-d. Similar to the H-P filtered AR(1), the medium long cycles (6-16 years) dominate and the three-quarter cycle is easily detected. Output and investment have most of the power around 8 year cycles, but consumption and inventory stock have a concentration of power at somewhat longer cycles.

The estimated spectra from US data (dashed curves) in Figures 2a-d are generally within the confidence intervals, as could be expected after H-P filtering. However, the estimated spectra tend to be more concentrated around the traditional business cycle frequencies ( 3 to 8 years) and to peak at shorter cycles than the model. In terms of the asymptotic distributions, these tendencies are significant (at the 10\% level) only for inventory stock, but in terms of the simulated small-sample distributions also for output and investment. This result is similar in spirit to that of Watson (1993). Finally, the three-quarter cycle is not found in the data, and the estimated spectra are clearly outside the $90 \%$ confidence bands.

The squared model coherencies of output and each of consumption, investment and inventory stock are, in a similar way, located approximately in the middle of the confidence intervals in Figures 3a-c. The coherencies, and also the phase shifts discussed below, are unaffected by the H-P filter. The model coherencies are very high for long and medium long cycles, and there is a common three-quarter cycle for output, investment and inventory stock. Figures 3a-c show that the estimated coherencies are generally below the lower boundaries of the $90 \%$ confidence intervals, at least for all cycles longer than three quarters. For the three-quarter cycle, there is some weak evidence in favor of a common cycle in output and investment, but not in output and inventory stock. The fit of the coherency of output and consumption is very bad for all cycle lengths; in fact, the estimated coherency is below the minimum of the 3000 simulations for almost all frequencies.

\footnotetext{
${ }^{6}$ The estimators employ a Bartlett-Priestley spectral window. Spectra, coherencies and phase shifts are calculated by weighting 19,13 , and 13 periodogram values respectively. The window and the asymptotic confidence intervals are discussed in Priestley (1981).
} 
As before, the model phase shifts are not shown, but are located approximately in the middle of the very narrow confidence intervals in Figures 4a-c. The most interesting features are that, at the business cycle frequencies, consumption and inventory stock lag output, while investment is contemporaneous. In contrast, the empirical phase shifts show that consumption is contemporaneous with output and that investment leads somewhat over output.

\subsection{Back to the time domain}

The frequency domain results have important implications for correlations. Figures $5 a$ - $d$ show the theoretical $90 \%$ confidence intervals for correlations with current output (solid curves) and estimates from the data (dashed curves). ${ }^{7}$ As before, the model correlations are (now exactly) in the middle of the confidence intervals and the small panels show significance levels for rejection of the model fit, based on simulations.

The spectra suggested that the business cycles in data are shorter than in the model. In Figures 5a-d this shows up in the form of clearly marked but fairly short cycles in the correlation functions: all correlations show peaks around \pm 6 years. The model correlation functions have longer cycles. As in the case with the spectra, the rejection of the fit is clearer in terms of the small-sample distribution.

In the estimated phase shifts, output does not lead consumption and investment in the way predicted by the model. This carries over directly to the correlations. The estimated correlations between current output and consumption lagged 2-6 quarters are clearly above the confidence interval. This makes the estimated correlation function almost symmetric around lag zero. Similarly, the estimated correlations between current output and investment, lagged once and twice, are also outside the confidence interval, which makes investment lead slightly over output.

Both coherencies and spectra suggest that the three-quarter cycle has only weak empirical support. In the time domain, this cycle shows up in the form of a zig-zag pattern for both auto- and cross-correlations for the model. ${ }^{8}$ Not surprisingly, data show no zig-zag pattern for correlations.

\section{Summary}

In this paper I have discussed an alternative way to test Kydland and Prescott's (1988) real business cycle model. The tests employ straightforward time-series methods to generate theoretical asymptotic distributions for frequency and time domain statistics, and also Monte-Carlo simulations to generate small-sample

\footnotetext{
${ }^{7}$ The confidence intervals are obtained by evaluating the inverse Fourier transform of the theoretical spectral density function $f(\lambda)$ to get theoretical correlations, and then using Bartlett's formula.

${ }^{8}$ The time domain aspects of the time-to-build feature are also discussed in Rouwenhorst (1991).
} 
distributions. The frequency domain statistics reveal certain model features which are hard to discover and test in the time domain, and they are in some cases also invariant to the controversial Hodrick-Prescott filter. The main discrepancies between the model and the data are the following. First, the data has shorter business cycles. Second, the data shows less comovements of different macro variables on the traditional business cycle frequencies. Third, and finally, the data shows a smaller leading role for output over the business cycle.

\section{References}

Altuğ, S. (1989), "Time-to-Build and Aggregate Fluctuations: Some New Evidence," International Economic Review, 30, 889-920.

Christiano, L.J. (1988), "Why Does Inventory Investment Fluctuate so Much?", Journal of Monetary Economics, 21, 247-280.

Cogley, T. and J.M. Nason (1992), "Effects of the Hodrick-Prescott Filter on Integrated Time Series," mimeo Haas School of Business, UCLA at Berkeley.

Harvey A.C. and A. Jaeger (1993), "Detrending, Stylized Facts and the Business Cycle," Journal of Applied Econometrics, 8, 231-247.

King, R.G. and S.T. Rebelo (1993), "Low Frequency Filtering and Real Business Cycles," Journal of Economic Dynamics and Control, 17, 207-231.

Kydland, F.E. and E.C. Prescott (1988), "The Workweek of Capital and Its Cyclical Implications," Journal of Monetary Economics, 21, 343-360.

Priestley, M.B. (1981), Spectral Analysis and Time Series, Academic Press.

Rouwenhorst, K.G. (1991), "Time to Build and Aggregate Fluctuations: A Reconsideration," Journal of Monetary Economics, 27, 241-254.

Singleton, K.J. (1988), "Economic Issues in the Analysis of Equilibrium Business Cycles," Journal of Monetary Economics, 21, 361-386.

Watson, M.W. (1993), "Measure of Fit for Calibrated Models," Journal of Political Economy, 101, 1011-1041. 


\section{Figure legends}

- Figure 1. Normalized spectral density of the AR(1) productivity shock before (thick solid) and after (dashed) H-P filtering, and the squared transfer function of the H-P filter (thin solid).

- Figures 2a-d. Normalized spectral density. Large panels: Point estimate from data (dashed) and theoretical 90\% confidence interval (solid) of the model economy. Small panels: simulated significance values for point estimates being equal to model economy.

- Figures 3a-d. Squared coherency. See Figures 2a-d for details.

- Figure 4a-d. Phase shift. See Figures 2a-d for details.

- Figure 5a-d. Correlation with current output. See Figures 2a-d for details. 


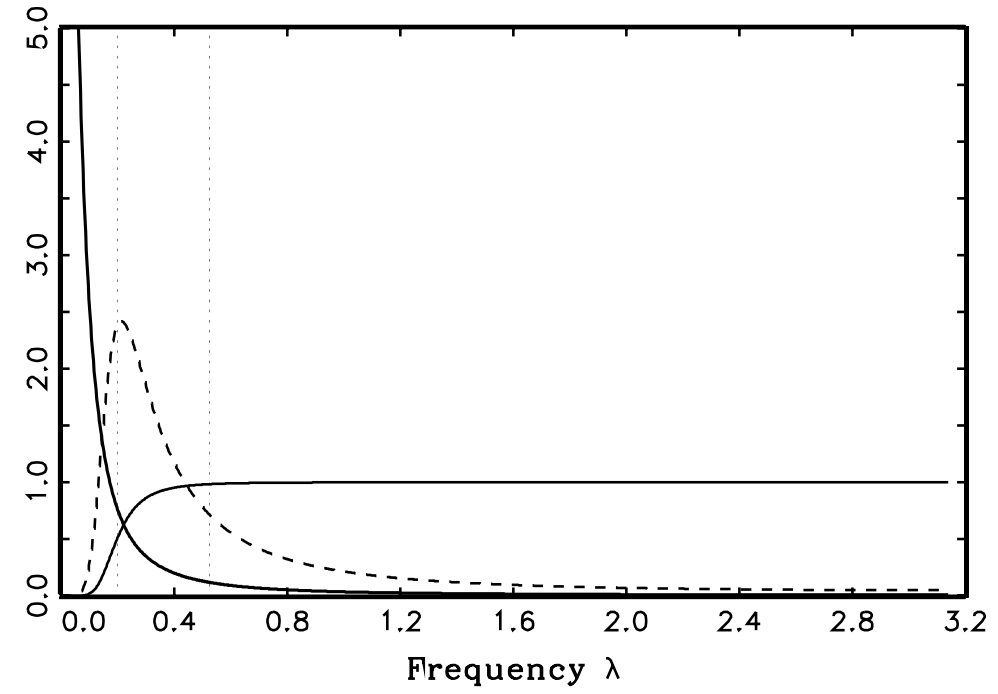


Output and Consumption

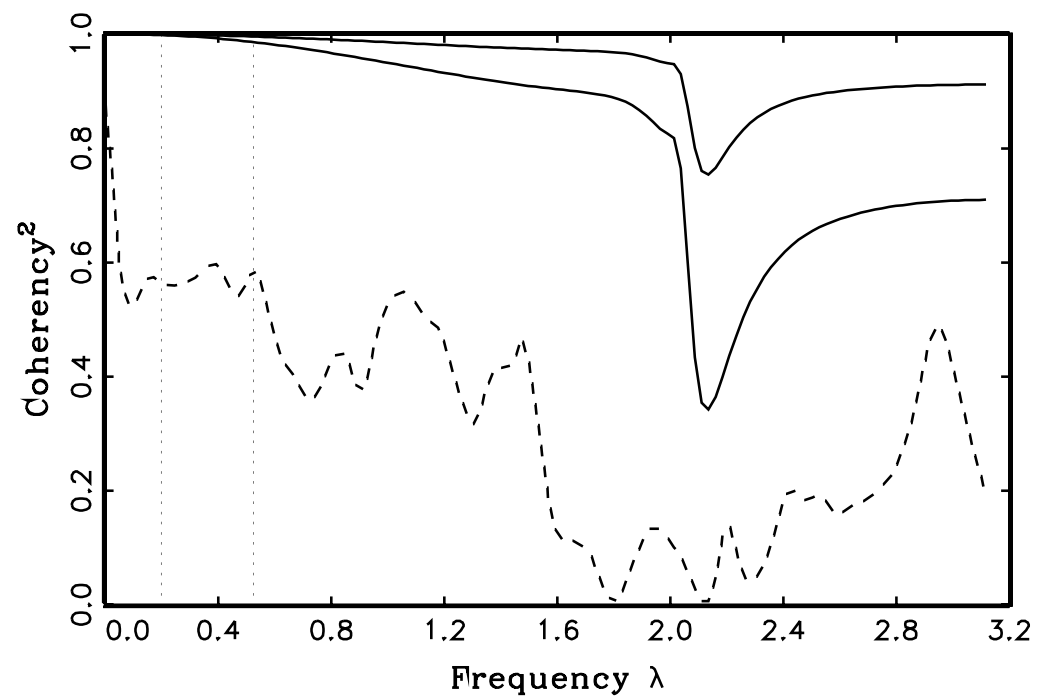

Output and Inventory Stock

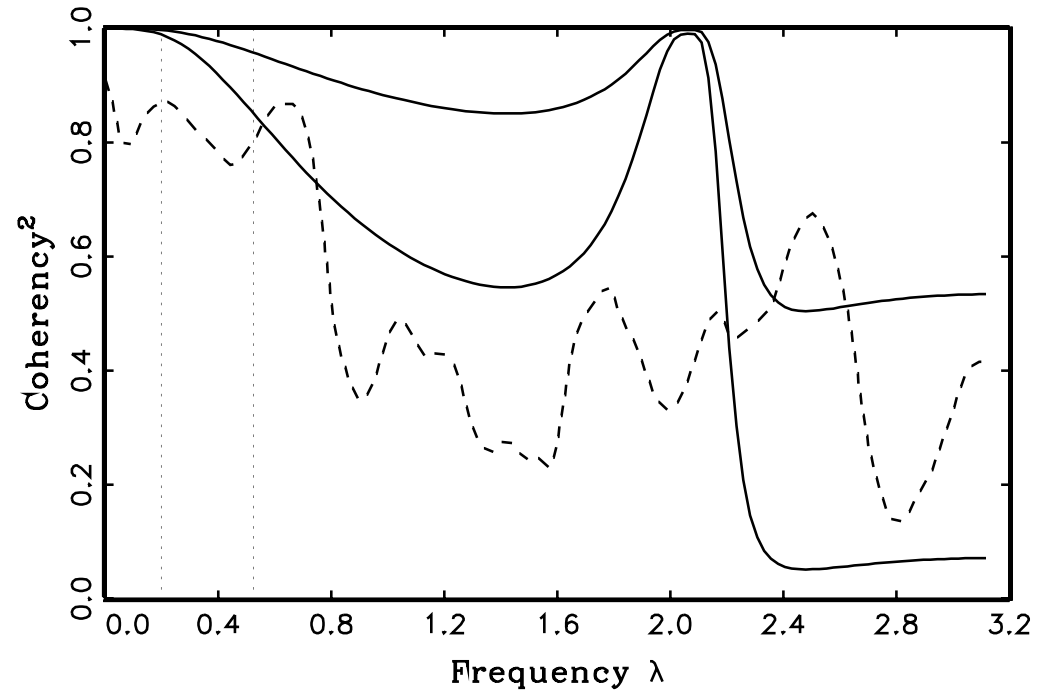

Output and Investment

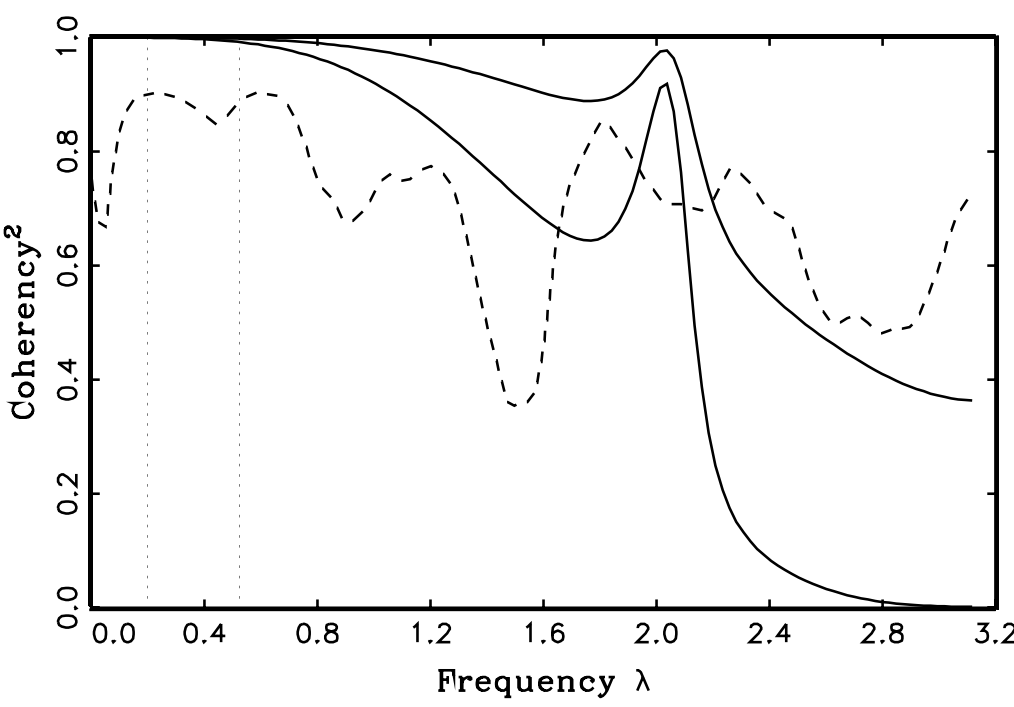

Significance levels in \%

Output and donsumption

Output and Investment
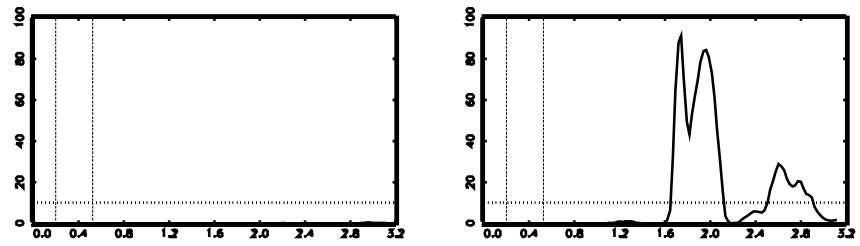

Output and Inventory Stock

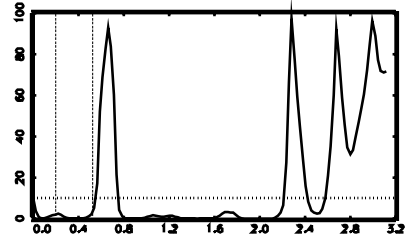


Output and Consumption

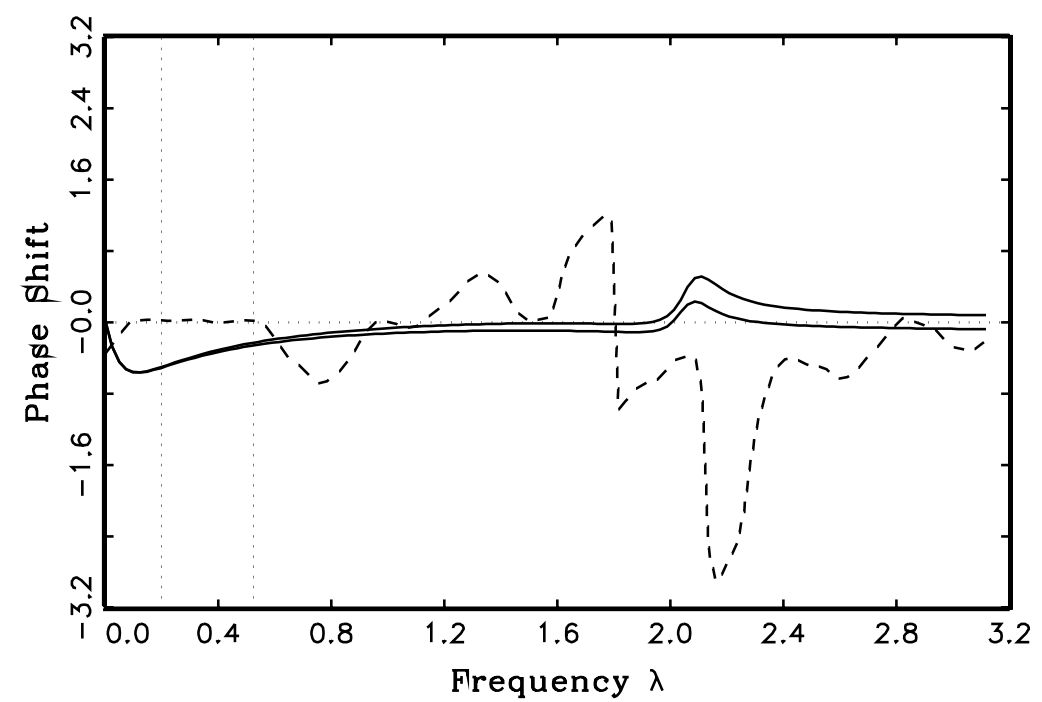

Output and Inventory Stock

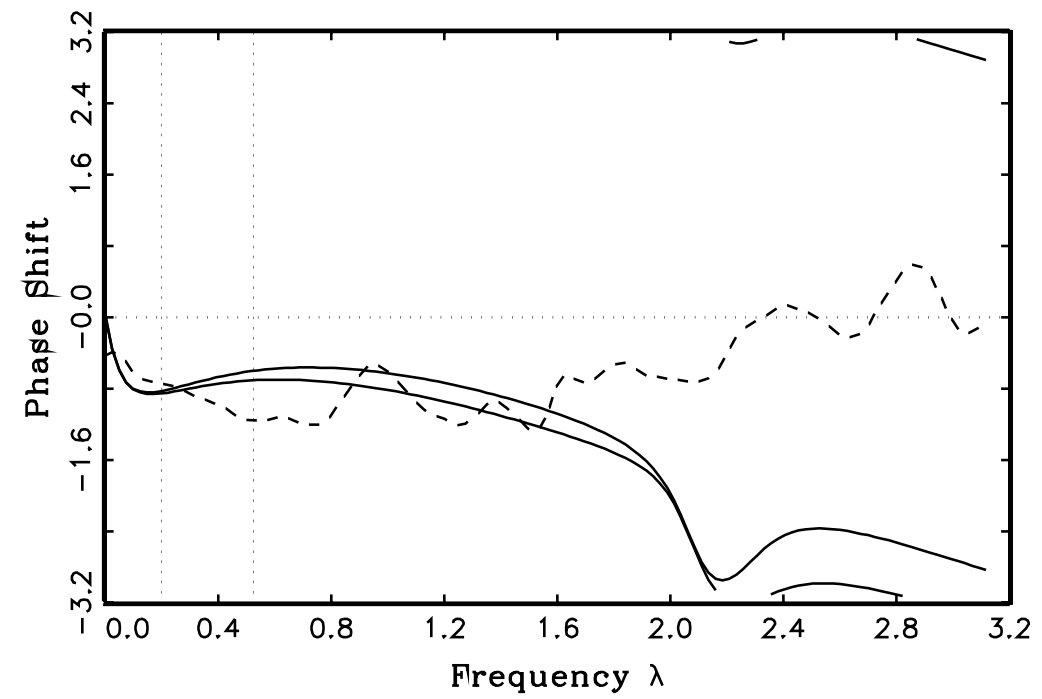

Output and Investment

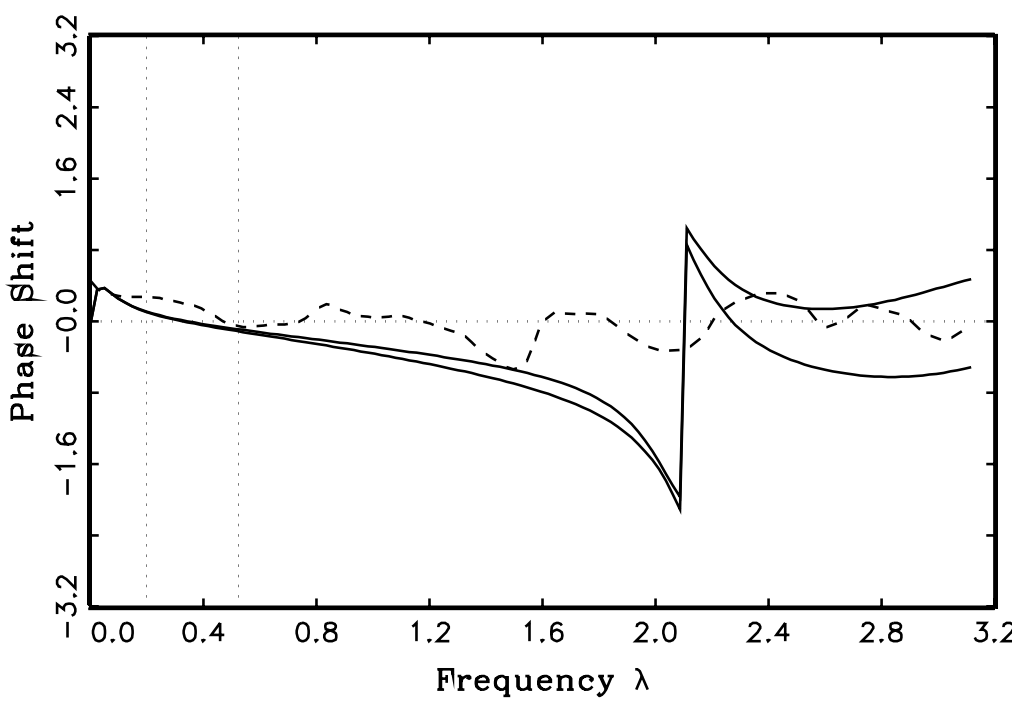

Significance levels in \%

Output and donsumption

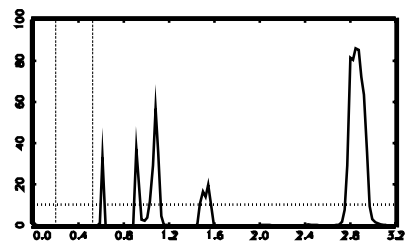

Output and Investment

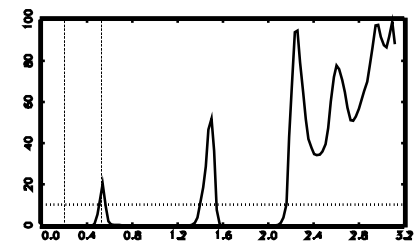

Output and Inventory Stock

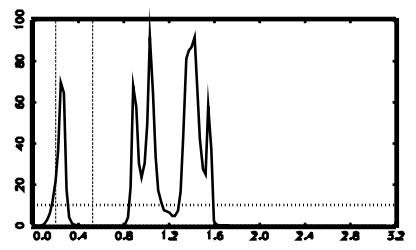


Output

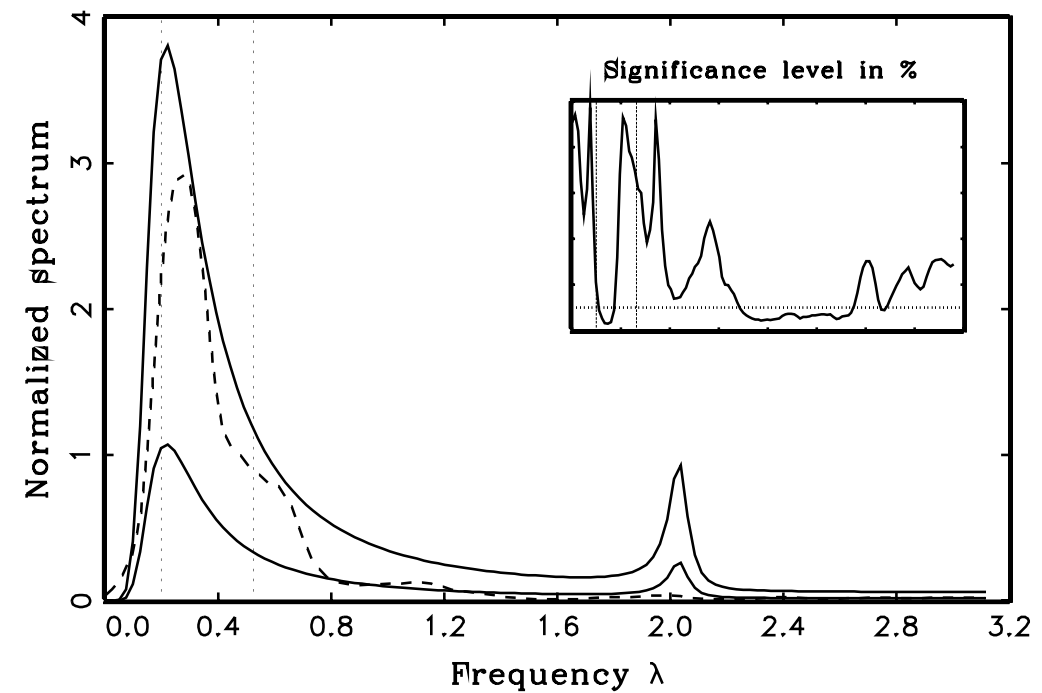

Investment

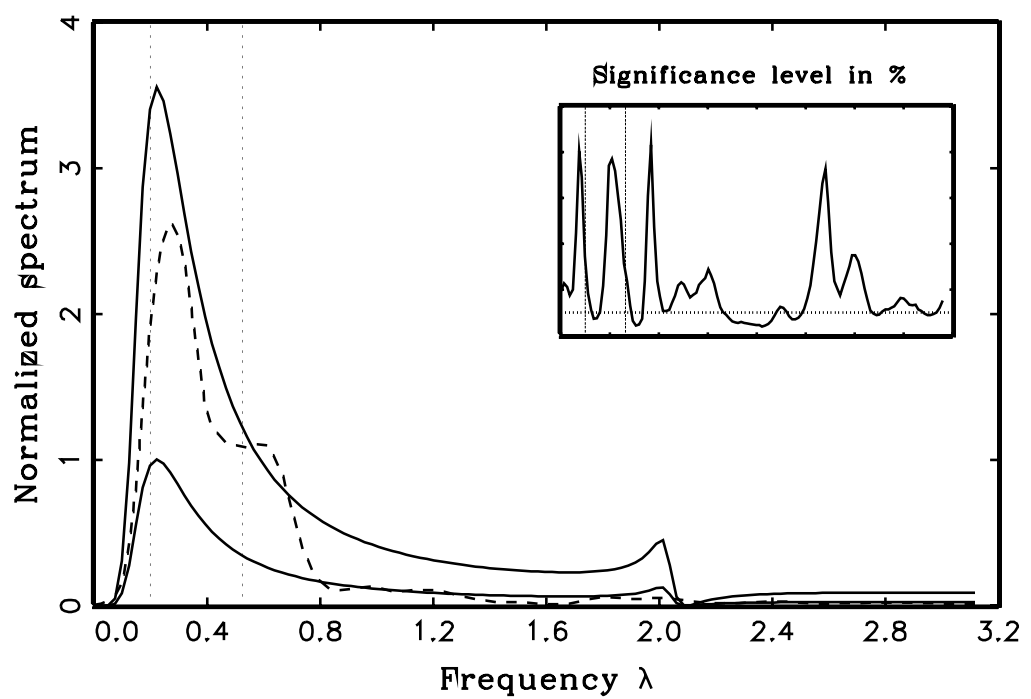

Consumption

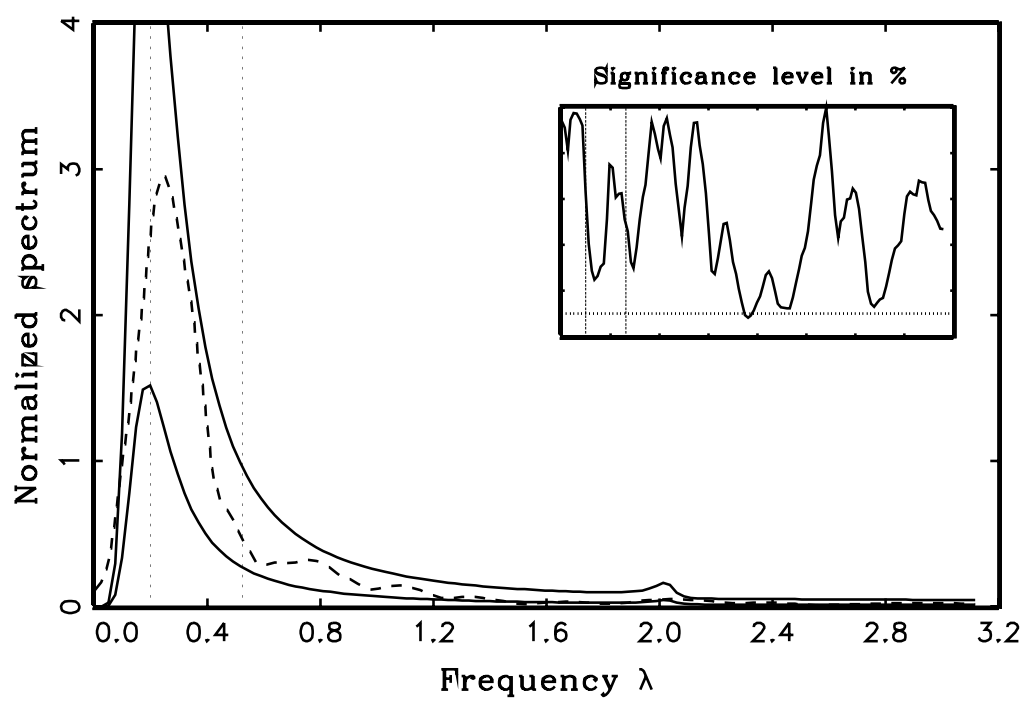

Inventory stock

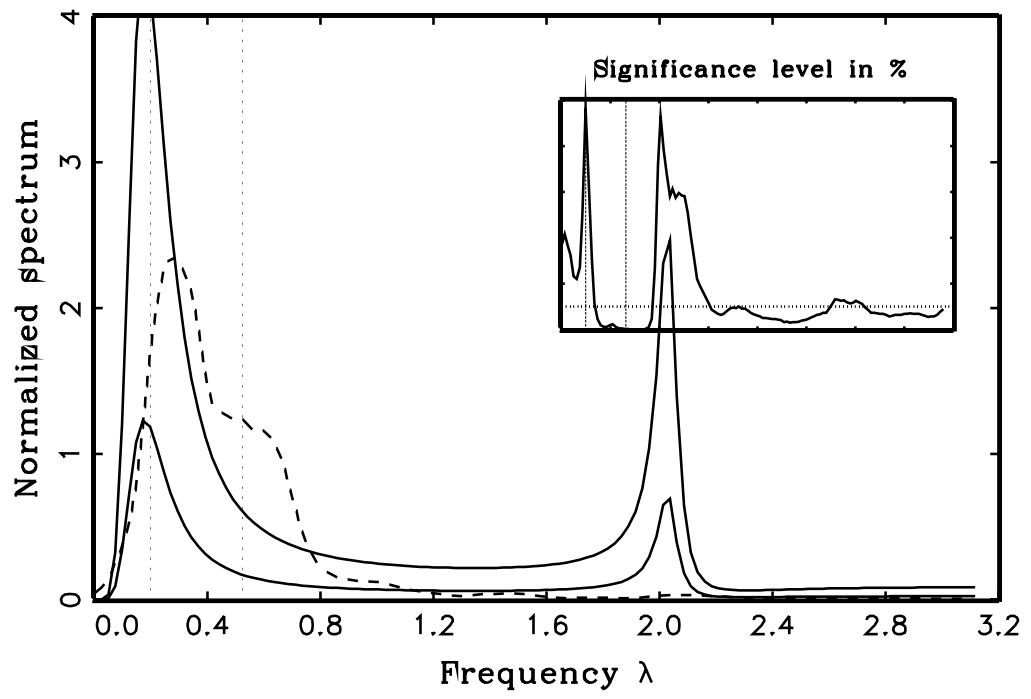

